\title{
Molecular characterization of HEXOKINASE1 in plant innate immunity
}

\author{
Wu Jing ${ }^{1 \dagger}$, Shahab Uddin ${ }^{1,2+}$, Rupak Chakraborty ${ }^{1}$, Duong Thu Van Anh ${ }^{1}$, Donah Mary Macoy ${ }^{1}$, Si On Park', \\ Gyeong Ryul Ryu', Young Hun Kim', Joon-Yung Cha², Woe-Yeon Kim² and Min Gab Kim ${ }^{1 *}$
}

\begin{abstract}
Hexokinase1 (HXK1) is an Arabidopsis glucose sensor that has a variety of roles during plant growth and devlopment, including during germination, flowering, and senescence. HXK1 also acts as a positive regulator of plant immune responses. Previous research suggested that HXK1 might influence plant immune responses via responses to glucose. Plant immune responses are governed by two main pathways: PAMP-triggered immunity (PTI) and effectortriggered immunity (ETI). PTI involves the recognition of Pathogen-Associated Molecular Patterns (PAMPs) and leads to increased callose formation and accumulation of pathogenesis response (PR) proteins. ETI acts in response to effectors secreted by Gram-negative bacteria. During ETI, the membrane-localized protein RPM1-interacting protein 4 (RIN4) becomes phosphorylated in reponse to interactions with effectors and mediates the downstream response. In this study, the effects of glucose on plant immune responses against infection with Pseudomonas syringae pv. tomato DC3000 and other P. syringae strains were investigated in the presence and absence of HXK1. Infiltration of leaves with glucose prior to infection led to decreases in bacterial populations and reductions in disease symptoms in wild-type Arabidopsis plants, indicating that glucose plays a role in plant immunity. Both PTI and ETI responses were affected. However, these effects were not observed in a $h \times k 1$ mutant, indicating that the effects of glucose on plant immune responses were mediated by HXK1-related pathways.
\end{abstract}

Keywords: Effector-triggered immunity, Glucose, Hexokinase, PAMP-triggered immunity, Plant immunity

\section{Introduction}

Sugar metabolism in plants is a critical and complex process that involves glycolysis, the tricarboxylic acid (TCA) cycle, and pentose phosphate pathways. Hexokinases (HXK) play key roles in sugar metabolism through phosphorylation of glucose to glucose-6-phosphate. The HXK family contains six members: HXK1, HXK2, HXK3, HKL1, HKL2, and HKL3 [1]. HXK1 is a multifunctional protein that is involved in sugar metabolism and signalling. An Arabidopsis $h x k 1$ mutant exhibited delayed flowering and senescence as well as smaller leaves and

\footnotetext{
*Correspondence: mgk1284@gnu.ac.kr

${ }^{\dagger}$ Wu Jing and Shahab Uddin contributed equally to this work

${ }^{1}$ College of Pharmacy and Research Institute of Pharmaceutical Science, PMBBRC, Gyeongsang National University, Jinju 660-701, Republic

of Korea

Full list of author information is available at the end of the article
}

root systems [2]. HXK1 was shown to affect the concentration of glucose in seedlings, and the absence of HXK1 significantly suppressed the effects of glucose [3].

Another HXK family member, HXK2, is involved in plant immunity. Overexpression of HXK2 led to enhanced plant resistance to pathogens and was correlated with elevated $\mathrm{H}_{2} \mathrm{O}_{2}$ production and expression of defensive genes [4]. HXK1 and HXK2 share similar features: both proteins have several functions, one of which is sugar-sensing in Arabidopsis [5].

Plants activate their innate immune systems via two pathways [6]. The first line of activation is PAMP-triggered immunity (PTI), which involves the recognition of Pathogen-Associated Molecular Patterns (PAMPs) by membrane-localized Pattern Recognition Receptors (PRRs) [7]. In the very early stages of the PTI response (within 1-5 min), PAMPs such as flagellin and EF-Tu are activated by PRRs, namely FLS2 and EFR [8, 9], after 
which the FLS-BAK1 complex forms within 2 min [10, 11]. Ion fluxes, oxidative bursts, and protein phosphorylation also occur during this stage. In the next stage of the response (5-30 $\mathrm{min})$, PTI induces ethylene biosynthesis, receptor endocytosis, and gene activation. These early responses lead to callose deposition and seedling growth inhibition over a longer timescale (hours-days) [12]. Subsequently, the accumulation of PR proteins instigates Systemic Acquired Resistance (SAR), which expands the local immune response of the plant to act against a broad spectrum of pathogens [13].

Effector triggered immunity (ETI) is the second line of activation of the plant immune system [6]. ETI acts in response to effector proteins secreted by the type III secretion system (TTSS) in Gram-negative plant-pathogenic bacteria [14]. Receptor (R) proteins which contain both Nucleotide-Binding (NB) and Leucine-Rich Repeat (LRR) domains can be triggered by direct interactions with their corresponding avirulence (Avr) effectors [15, 16] or indirectly via detection of the action of an $A v r$ effector on its target $[17,18]$.

The hypersensitive response (HR), a type of plant resistance response, induces programmed cell death at infection sites and inhibits pathogen growth [19]. Arabidopsis protein RIN4 is a well-characterized component of this type of resistance response, and can be explained by the guard hypothesis [6]. RIN4 is a small protein that localizes to the plasma membrane alongside several guard proteins, including RPM1 (resistance to $P$. syringae pv. maculicola 1) and RPS2 (resistance to P. syringae 2) [20, 21]. Bacterial type III effector protein AvrRpm1 acts via RIPK and related kinases to mediate phosphorylation of RIN4 and thereby activate RPM1 [22]. AvrRpt2 is a cysteine protease which cleaves RIN4 at two sites, producing three fragments of $15.9 \mathrm{kDa}, 6.4 \mathrm{kDa}$, and $1.2 \mathrm{kDa}$ [23]. Degradation of RIN4 activates RPS2 and may induce a conformational change in the RPS2-RIN4 complex [24].

The relationship between sugars such as glucose and innate plant immunities remain poorly understood. In this study, the effects of glucose on plant immunity in the presence and absence of HXK1 were assessed, and links to PTI and ETI mechanisms were elucidated.

\section{Materials and methods}

\section{Plant lines and growth conditions}

Arabidopsis thaliana accessions Columbia (Col-0) and Landsberg erecta (Ler) and HXK1 deficient mutant $h x k 1$ (SALK_034233) (in both the Col-0 and Ler backgrounds) were a kind gift from professor Woe Yeon Kim at the Division of Applied Life Science, Gyeongsang National University, Republic of Korea. Plants were cultivated in a growth chamber with a $16 \mathrm{~h}$ light / $8 \mathrm{~h}$ dark light cycle, light intensity $75 \mu \mathrm{mol} \mathrm{m} \mathrm{m}^{-2} \mathrm{~s}^{-1}$, humidity $85 \pm 1 \%$, and temperature $22 \pm 1{ }^{\circ} \mathrm{C}$. After 2 weeks, seedlings were transferred to a large tray and cultivated until seedlings were 4-5 weeks old. All seedlings were grown following long days (16 h light/8 $\mathrm{h}$ dark) to determine the protein accumulation and gene expression levels.

\section{Bacterial strains and treatment}

Pseudomonas syringae pv. tomato DC3000 containing an empty vector plasmid pVSP61(Pto), type III effector protein-expressing strains Pto AvrRpm1 and Pto AvrRpt2, Pto TTSS-deficient mutant $h r c C^{-}$, and P.syringae pv. Phaseolicola $(P p h)$ were provided by Professor David Mackey's Lab, Ohio State University U.S.A. Bacterial strains were grown at $27{ }^{\circ} \mathrm{C}$ for 2 days in King's broth medium containing appropriate antibiotics. WT and $h x k 1$ plants were evenly assigned into either the Mock group infiltrate with water $24 \mathrm{~h}$ before inoculating with $10 \mathrm{mM} \mathrm{MgSO}$ ), the Mock + bacteria (Pto, P.syringae pv. Phaseolicola (Pph) or Pto AvrRpm1 and Pto AvrRpt2) infiltrate with water $24 \mathrm{~h}$ before inoculating bacteria, or the glu + bacteria group inoculated with $2.5 \%$ glucose 24 before inoculating with bacteria. Growth and symptom analysis of Pto DC3000 were conducted as described in [25]. Bacterial solutions were syringe inoculated into 4 to 5 weeks old leaves. Leaf, discs were ground to homogeneity in $10 \mathrm{mM} \mathrm{MgCl}_{2}$ for all growth experiments, and the titer determined by serial dilution and plating.

\section{Callose staining}

Four-week-old leaves were syringe-infiltrated with $100 \mu \mathrm{M}$ flg22 and distilled water or pretreated with water or glucose $24 \mathrm{~h}$ before inoculating with flg22 or water as a control and collected after $16 \mathrm{~h}$ and stained with methyl blue followed [25], and mounted in 50\% glycerol, and examined by fluorescence microscopy (OPTICA, Ponteranica BG Italy). Representative views of these pictures were randomized.

\section{Western analysis}

Western blot was executed as previously mentioned with little modification [26]. Approximately $100 \mu \mathrm{g}$ plant tissue was extracted by mixing with $100 \mu \mathrm{l}$ protein extraction buffer (100 mM Tris- $\mathrm{HCl}(\mathrm{pH} 7.5), 150 \mathrm{mM} \mathrm{NaCl}$, $1 \mathrm{mM}$ EDTA, 0.5\% NP-40, $5 \mathrm{mM}$ DTT, and plant protease inhibitor cocktail) and centrifuging at 13,000 rpm for $10 \mathrm{~min}$ at $4{ }^{\circ} \mathrm{C}$. Proteins were quantified by bicinchoninic acid assay. Proteins were separated on a 12\% SDS-PAGE gel (Mini-protein, Bio-Rad) and transferred to polyvinylidenefluoride membrane. Anti-PR-1 sera were used at a 1:2000 dilution. Chemiluminescent detection and band quantification were performed using a ChemiDoc XRS system (Bio-Rad). 


\section{RNA analysis}

Total RNA was extracted from approximately $100 \mu \mathrm{g}$ plant tissue using an RNA extraction kit (QIAGEN), after which RNA was treated with DNaseI. RNA was quantified using a Nanodrop spectrophotometer (DeNovix). Total RNA (approximately $1 \mu \mathrm{g}$ ) was used for cDNA synthesis (ENZYMOMICS). Real-time PCR was performed using a Bio-Rad Real-Time PCR detection system with SYBR Green Super Mix (Cosmo Genetech). Ribosomal protein was used as a control. The following primers were used: FRK1, TCAGAGATC GCTCTTGCTTGTA and CTGTAAGCATTTTCG TCGAGTC; WRKY29, AAGGATCTCCATACCCAA GGA and TTATGGTGAATTTCTCCGGG; Ribosomal protein, CGGACAATTTGGATTCGTTG and ACC ACCACCGGAGTATCTCG. Three biological and two technical replications were conducted.

\section{Ion leakage and HR assay}

For the ion leakage measurement as previously described [18], 12 leaf discs were collected immediately after inoculation with $2 \times 10^{8} \mathrm{CFU} / \mathrm{ml}$ bacterial strains Pto expressing AvrRpm 1 and AvrRpt2 and washed with $50 \mathrm{ml}$ of $\mathrm{dd}_{2} \mathrm{O}$, after $30 \mathrm{~min}$ leaf discs were re-sus-

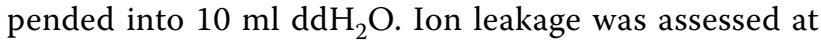
different time points. For HR assay WT and $h x k 1$ plants were pretreated with water as a control and $2.5 \%$ glucose 24 h before inoculating with $2 \times 10^{8} \mathrm{CFU} / \mathrm{ml}$ bacterial strains Pto expressing AvrRpm 1 and AvrRpt2 and $10 \mathrm{mM} \mathrm{MgCl}_{2}$ as control.

\section{Results}

HXK1 positively enhances plant defenses against pathogen infection

To investigate the role of HXK1 in PTI and ETI, the plant immunity-related functions of HXK1 were evaluated using HXK1 deficient mutants $(h x k 1)$ generated in the Arabidopsis Col-0 and Ler backgrounds. Five-weekold plants were inoculated with Pseudomonas syringae pv. tomato DC3000 (Pto). Typical disease symptoms appeared in inoculated leaves 4 days after inoculation [27]. Both $h x k 1$ mutants exhibited more severe disease symptoms than the corresponding wild-type (WT) plants (Fig. 1a, c). Bacterial populations in the infected $h x k 1$ mutants were higher than in WT, consistent with the observed infection phenotype (Fig. 1b, d). These results indicate that $\mathrm{HXK1}$ might play an important role in plant defense, and that absence of HXK1 might negatively affect plant immunity to bacteria, resulting in more severe disease symptoms.
HXK1 plays a central role in PAMP-triggered immunity

Previous research showed that expression of FRK1 and WRKY29, two PAMP-response marker genes, was induced by flg22 [28]. To investigate the role of HXK1 in PTI, thus, we found that flg22 was treated in $h x k 1$ mutant plants and wild type. Lower amounts of FRK1 and WRKY29 transcripts accumulated in a $h x k 1$ mutant line than in WT (Fig. 2a, b). These results suggest that HXK1 is required for flg22-induced gene expression. Similarly, when $h x k 1$ mutant plants were infected with the non-pathogenic $P$. syringae $h r c C^{-}$mutant, which lacked a functional type III secretion apparatus, bacterial accumulation was higher in $h x k 1$ mutant plants compared to WT (Fig. 2c), indicating a positive role for HXK1 in PTI. Callose formation in plants is a defensive response that involves the deposition of polysaccharides to reduce the number of microorganisms that can enter the plant cell $[29,30]$. Flg22-induced callose deposition was significantly reduced in $h x k 1$ mutants compared to WT (Fig. 2d).

PR1 can be used as a marker for PTI due to its role in blocking $\beta-1,3$ glucanase accumulation [31]. PR1 is expressed by $P$. syringae pv. phaseolicola (Pto Pph), a nonhost pathogen [32]. PR1 expression by $P p h$ was reduced in $h x k 1$ mutants compared to Col-0 (Fig. 2e), suggesting that the absence of HXK1 reduced susceptibility to bacteria in Arabidopsis through reductions in callose deposition and PR1 accumulation.

\section{HXK1 partly enhances plant immunity through effector-triggered immunity}

Two different effectors were used to assess the role of HXK1 in effector-triggered immunity: avirulent P.syringae strains Pto AvrRpm1 and Pto AvrRpm2. RPM1 is a resistance protein that recognizes phosphorylated RIN4, leading to the hypersensitive response. RIN4 becomes phosphorylated in response to infiltration of Pto AvrRpm1. Infiltration of Pto AvrRpm2 leads to degradation of RIN4, which is recognized by another plant resistance protein, RPS2, leading to HR [26, 33].

In bacterial growth assays, Pto AvrRpm1 proliferation was higher in the $h x k 1$ mutant than in WT (Fig. 3a), suggesting that a lack of HXK1 impaired the response to bacterial infection in Arabidopsis. However, no significant differences were found in Pto AvrRpt2 proliferation between $h x k 1$ and WT (Additional file 1: Fig. S1a). Inoculation with Pto AvrRpm1 stimulated HR in WT at approximately $5 \mathrm{~h}$-post-infection (hpi), but this response was delayed in the $h x k 1$ mutant (Fig. 3b). HR was observed at approximately $9 \mathrm{hpi}$ in both $h x k 1$ and WT leaves inoculated with Pto AvrRpt2 (Additional file 1: Fig. S1b). Ion leakage from plant cells is characteristic of 

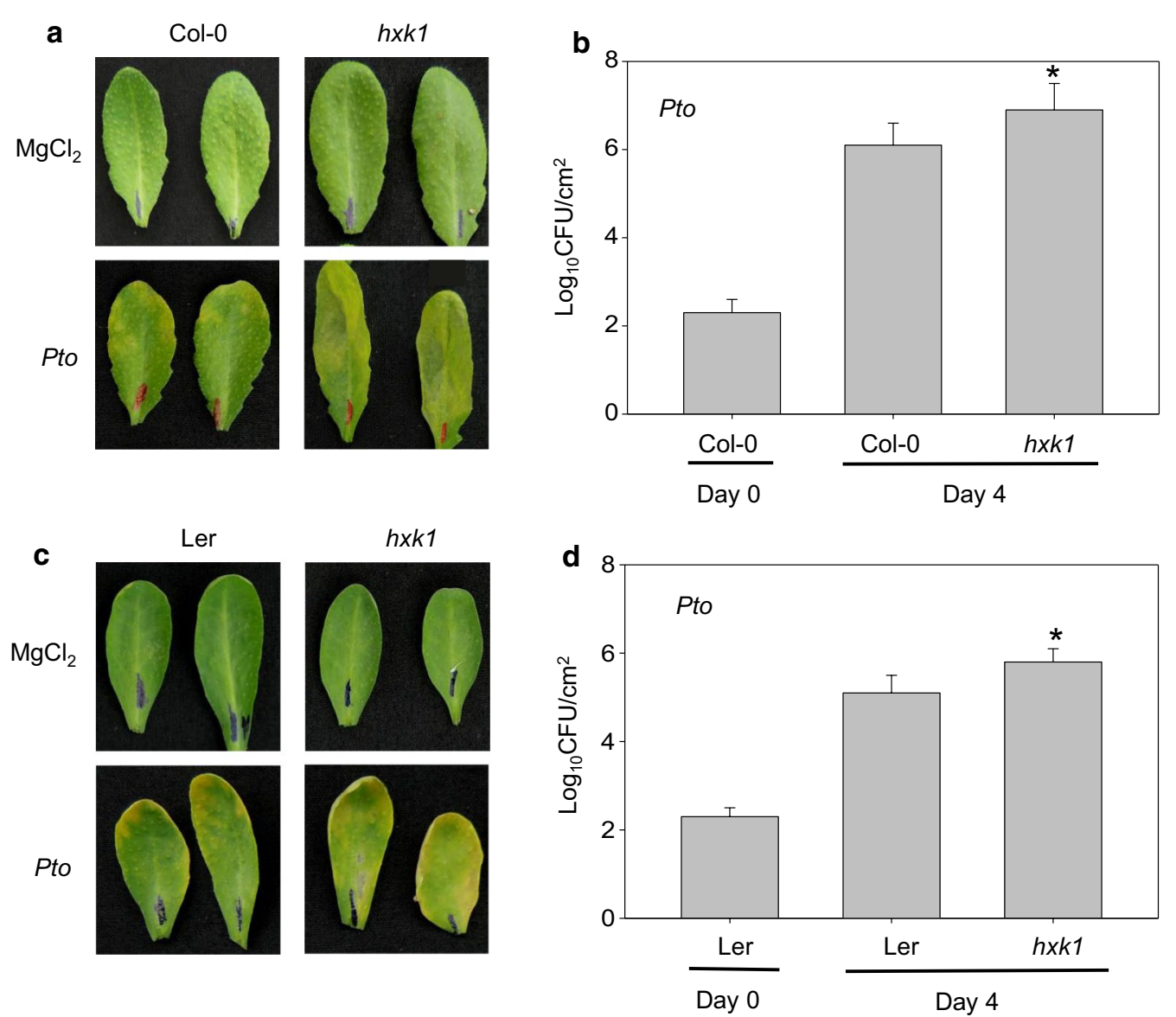

Fig. 1 HXK1 positively enhances plant defences against P. syringae pv. tomato DC3000 infection. a, c Leaves of WT and hxk1 seedlings (Col-0 and Ler background) after infection with P. syringae pv. tomato DC3000 (Pto) at $2 \times 10^{5} \mathrm{CFU} / \mathrm{ml}$ concentration or $\mathrm{MgCl}_{2}$ (control). b, d. Bacterial levels in $h \times k 1$ and WT plants after infections as $(\mathbf{a}, \mathbf{c})$. Error bars represent the standard error of the mean $(n=3)$. Asterisks indicate significant differences $(P<0.05$, Student's $t$-test). All experiments were performed three time

HR-mediated cell death, and an ion leakage assay was therefore used to assess cell death. A faster, more severe cell death response reduces the numbers of bacteria that can spread to neighboring plants tissues. As expected from the results of the HR assay, ion leakage was reduced in $h x k 1$ compared with WT after Pto AvrRpm1 infiltration (Fig. 3c), supporting the hypothesis that HXK1 is important for a rapid ETI response to occur. Taken together, our results indicate that HXK1 positively enhances plant defense against $P$. syringae pv tomato DC3000 infection through its involvement in PTI and its partial involvement in ETI.

\section{Exogenous glucose positively enhances plant immunity}

Sugars have multiple functions in plants. As well as its involvement in carbohydrate biosynthesis during photosynthesis and its role in respiration, previous research showed that low concentrations of glucose positively regulated root growth and development in Arabidopsis seedlings [34]. To investigate the role of glucose in plant immunity, different concentrations of glucose was infiltrated into the leaves of mature 5-week-old Arabidopsis plants. Shortly after infiltration, plants showed an HRlike reaction due to the osmotic pressure resulting from the addition of glucose. At lower glucose concentrations, the plants assimilated the exogenous glucose over the following $24 \mathrm{~h}$, releasing the osmotic stress and allowing leaves to return to a normal non-HR state. High concentrations of glucose (5\% and above) caused permanent damage to plants (Additional file 1: Fig. S2a). Tolerance to glucose was higher in mature plants than in seedlings, where infiltration with $1.5 \%$ or $3 \%$ glucose was sufficient to suppress seedling growth (Additional file 1: Fig. S2b) [2]. The direct damage caused to cells as a result of the osmotic stress imposed by high concentrations of glucose was irreversible.

Plant defense is not a unilateral process, but involves bilateral interactions between plants and pathogens. To 

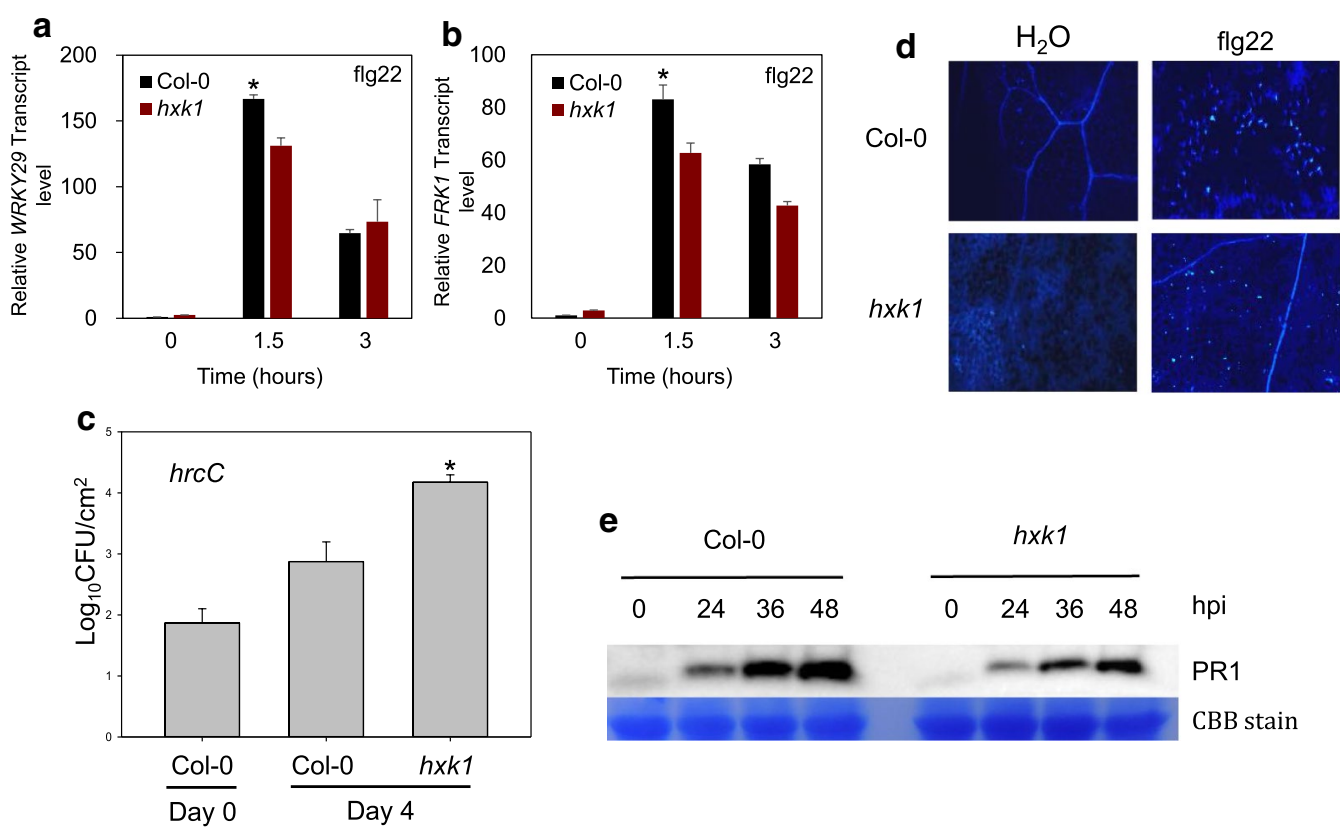

e Col-0 $h \times k 1$

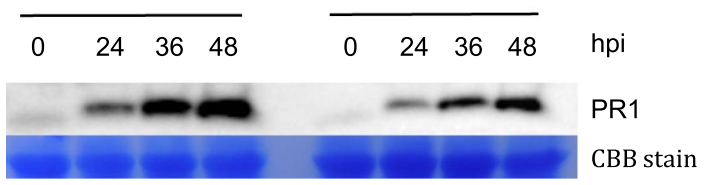

Fig. 2 HXK1 is involved in PTI. a, b Expression of WRKY29 and FRK1 mRNA in hxk1 mutant and WT Arabidopsis infiltrated with flg22. c Bacterial accumulation in Col-0 and $h \times k 1$ after inoculation with $2 \times 10^{5} \mathrm{CFU} / \mathrm{ml}$ of non-pathogenic $P$. syringae hrc $C^{-}$. Error bars represent the standard error of the mean $(n=3)$. The asterisk indicates a statistically significant difference $(P<0.05$, Student's $t$-test) $(\mathbf{a}-\mathbf{c})$. $\mathbf{d}$ Callose deposition in Col-0 and $h \times k 1$. e PR1 accumulation in Col-0 and $h \times k 1$ after inoculation with of $2 \times 10^{6} \mathrm{CFU} / \mathrm{ml}$ Pph. Coomassie Brilliant Blue (CBB) staining was used to ensure equivalent loading. hpi hours post infection. Similar results were obtained in three independent experiments

investigate the effects of glucose on bacteria, Pto, Pto AvrRpm1, and Pto AvrRpt2 were cultivated in liquid KB medium containing a range of glucose concentrations. Bacterial populations were assessed at various time points after exogenous glucose treatment, and the bacterial growth was impacted at approximately $16 \mathrm{~h}$ after treatment (Additional file 1: Fig. S3). After confirming that exogenous glucose suppressed bacterial growth in-vitro, similar experiments were performed to understand the effects of glucose on plant growth.

To assess the effects of bacteria on plants in the presence of glucose, low concentrations of glucose were pre-infiltrated into plants leaves followed by infiltration with Pto after 1 day. Disease symptoms (Fig. 4a) and bacterial growth (Fig. 4b) were reduced in the presence of glucose. These results suggest that plant immunity was enhanced by glucose, and that pre-infiltration with glucose may prime the immune response by activating pathways that allow the plant to react more rapidly and more vigorously to pathogen challenge. Howerver, the glucose benefit was lost in the $h x k 1$ mutant both with respect to disease symptoms (Fig. 4c) and bacterial growth (Fig. 4d). This suggests that the role of glucose in plant immunity is linked to the HXK1 pathway, but the specific pathways involved remain unknown.
Glucose positively regulates PAMP-triggered immunity via the HXK1 pathway

Callose deposition was examined in infiltrated WT leaves to assess the effects of glucose on PTI (Fig. 5a). Glucose induced higher levels of callose deposition and production of thicker plant cell walls compared to untreated controls. Thinner plant cell walls are more conducive to bacterial entry. Additional callose deposition was not observed in the $h x k 1$ mutant after treatment with exogenous glucose (Fig. 5b). PR1 accumulation is indicative of the speed and intensity of the PTI response. PR1 accumulation was increased and was more rapid in glucosetreated WT plants than in control plants (Fig. 5c), but no similar effect was seen in the $h x k 1$ mutant (Fig. 5d). Taken together, these results indicate that glucose positively regulates PTI via a HXK1-related pathway.

\section{HXK1 plays a key role in glucose up-regulation of effector-triggered immunity}

Effector proteins from Pto AvrRpm1 and Pto AvrRpt2 were used to assess the effects of glucose on ETI. As with PTI, pre-infiltration of glucose prior to bacterial infiltration successfully reduced bacterial growth in WT Arabidopsis exposed to Pto AvrRpm1 or Pto AvrRpt2 (Fig. 6a), but no reductions in bacterial growth or disease 

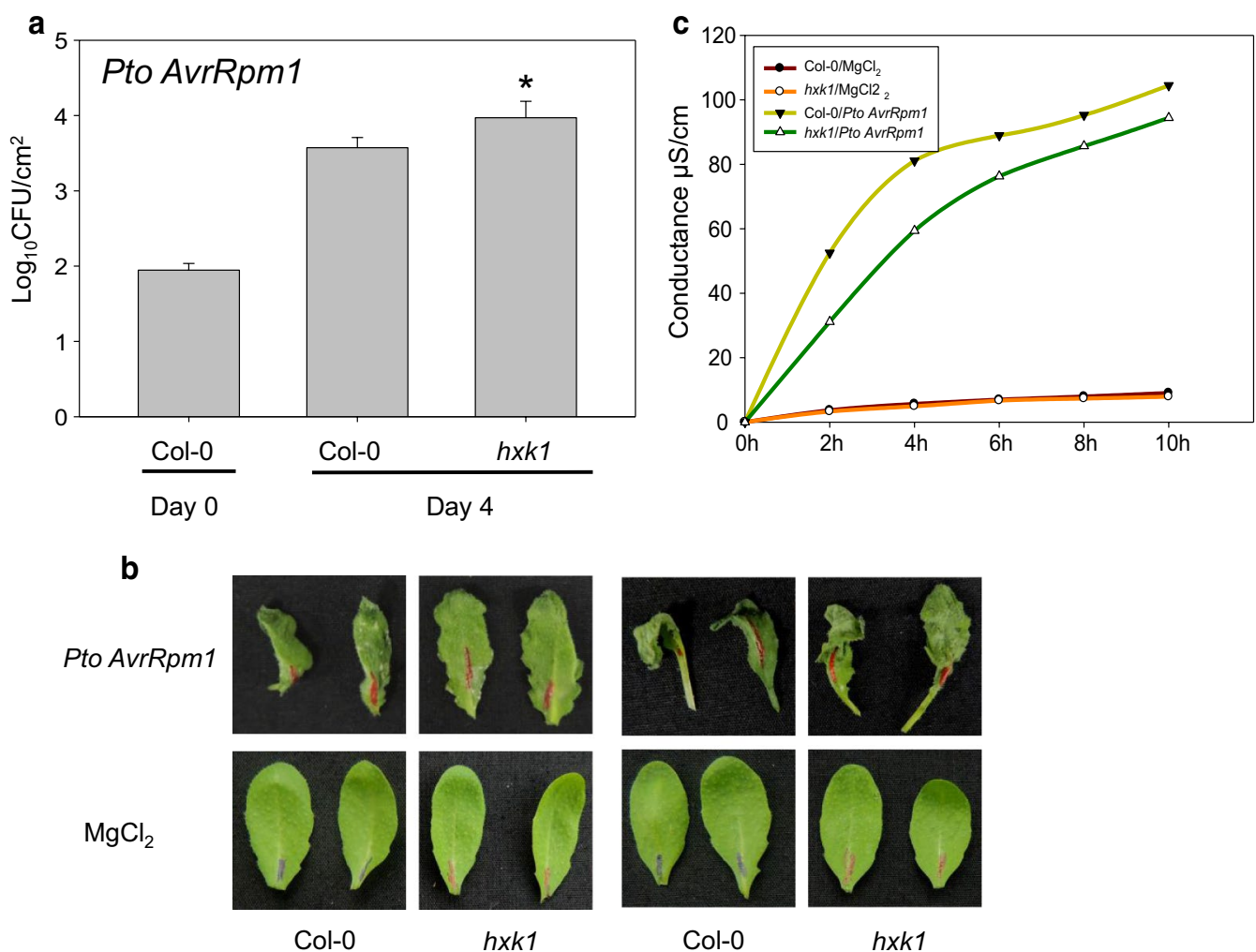

$5 \mathrm{~h}$
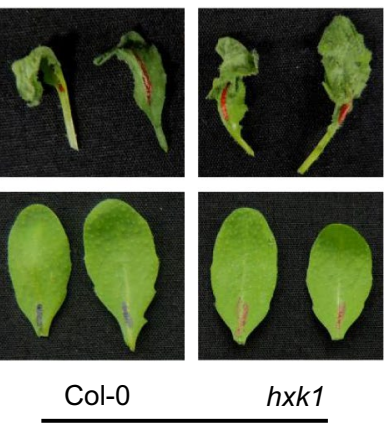

$7 \mathrm{~h}$

Fig. 3 HXK1 is required for RPM1-dependent suppression of Pto AvrRpm 1. a Bacterial accumulation in Col-0 and hxk1 after inoculation with $2 \times 10^{5} \mathrm{CFU} / \mathrm{ml}$ of Pto AvrRpm1. Error bars represent the standard error of the mean $(\mathrm{n}=3)$. The asterisk indicates a statistically significant difference $\left(P<0.05\right.$, Student's t-test). b lon leakage assay in $h \times k 1$ and WT leaves infiltrated with Pto AvrRpm 1 or $\mathrm{MgCl}_{2}$ (control). c Speed of HR in $h \times k 1$ and WT infiltrated with Pto AvrRpm1 or $\mathrm{MgCl}_{2}$ (control). Three independent experiments were conducted.

symptoms were seen in the $h x k 1$ mutant (Fig. 6b). This suggests that glucose activates RIN4-related pathways that enhance plant immunity through ETI. A hypersensitive response assay was performed to further elucidate the function of glucose in ETI. In Col-0, HR was observed soon after Pto AvrRpm1 and Pto AvrRpt2 infiltration following glucose pre-infiltration (Fig. 6c). However, glucose did not induce a faster HR response in the $h x k 1$ mutant (Fig. 6d). Rapid HR limits the number of bacteria that can spread to other parts of the plant and, through the induction of early localized cell death, glucose positively regulates ETI by HKX1-related pathways. Overall, glucose enhanced PTI and ETI after exposure to P. syringae pv. tomato DC3000 infection, and this effect was likely mediated by HKX1-related pathways.

\section{Discussion}

Exogenous sugar supplementation was previously shown to impact bacterial growth [35]. In this study, glucose supplementation suppressed bacterial growth in vitro in a largely dose-dependent manner Additional file 1: Fig. S3, consistent with previous research [36]. Low concentrations of glucose can enter bacterial cells and activate specific pathways that stop or decrease bacterial growth. High glucose concentrations may inhibit bacterial growth through osmotic pressure. Here, when exogenous glucose was added to bacterial growth media, the glucose concentration changed over time. As glucose concentrations within bacterial cells increased, the osmotic pressure within bacterial cells reduced with respect to the outside environment. Under these circumstances, glucose can be transported by carrier proteins and can then activate cellular pathways to slow bacterial growth. However, when the concentration of glucose outside the bacterial cells is too high, the bacteria experience osmotic stress. This results in dehydration, which also suppresses bacterial growth. It is possible that suppression of bacterial growth directly activates related glucose pathways in the absence of osmotic pressure. Additional research is 

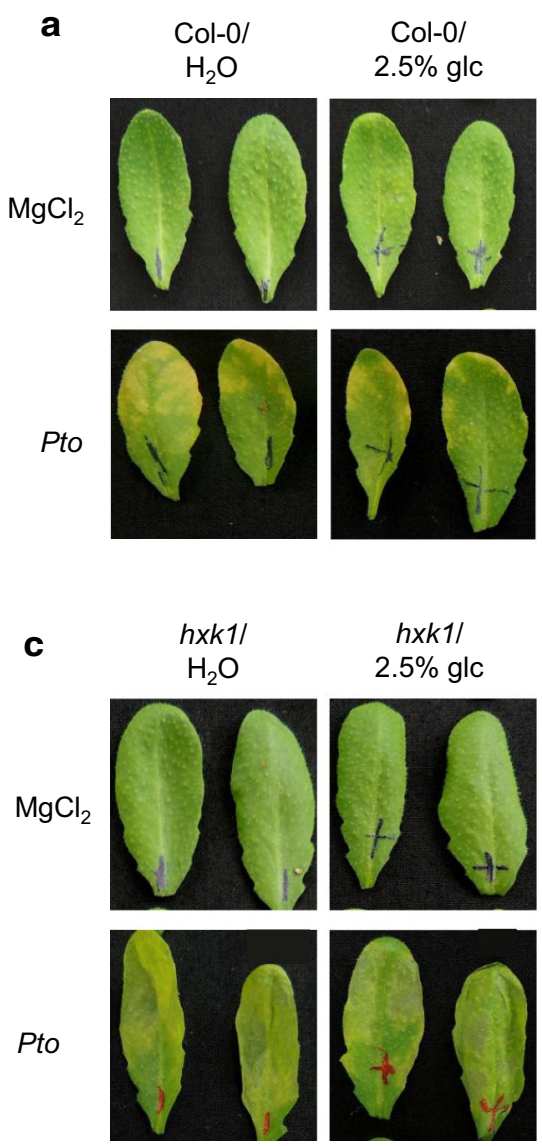
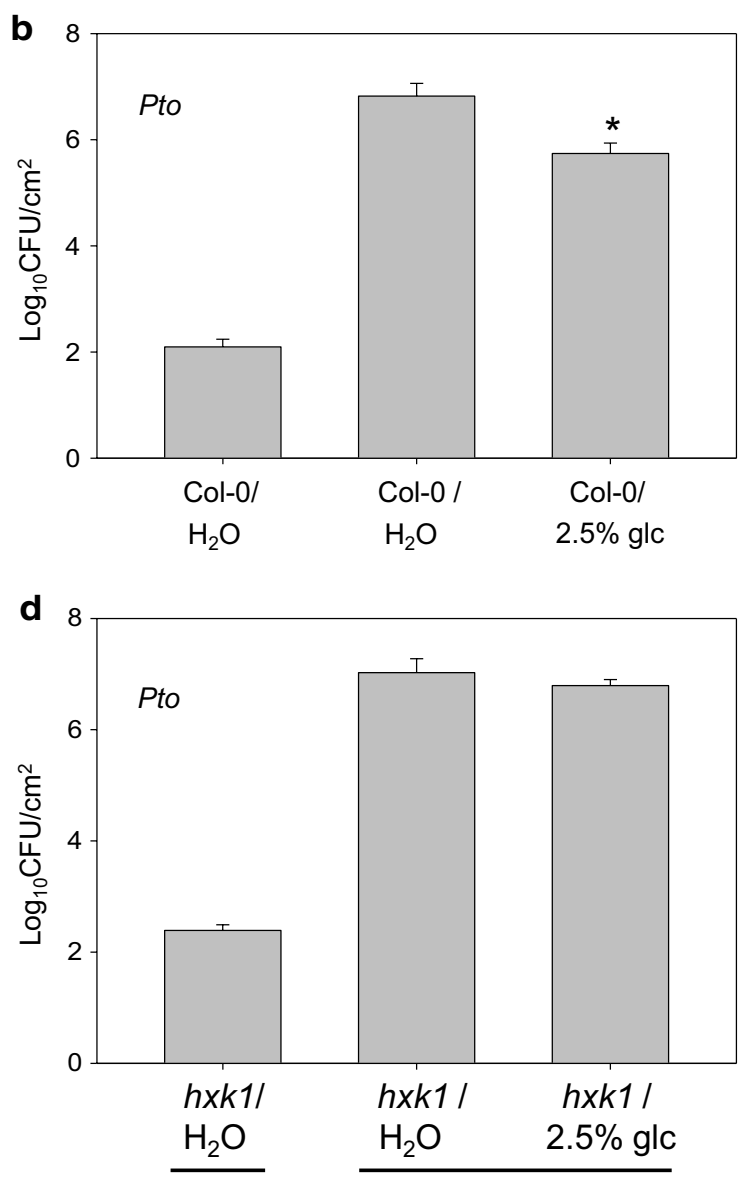

Day 0

Day 4

Fig. 4 Exogenous glucose positively enhances plant immunity against Pto. a, c Disease symptoms in Col-0 and $h \times k 1$ leaves infiltrated with $\mathrm{H}_{2} \mathrm{O}$ or 2.5\% glucose, followed 1 day later by infiltration with Pto $\left(2 \times 10^{6} \mathrm{CFU} / \mathrm{ml}\right)$ or $\mathrm{MgCl}_{2}$ (control). b, $\mathbf{d}$ Bacterial growth in leaves infiltrated as described in $(\mathbf{a}, \mathbf{c})$. Error bars represent the standard error of the mean $(n=3)$. The asterisk indicates a statistically significant difference $(P<0.05$, Student's

$t$-test). All experiments were conducted three time

required to further elucidate the effects of glucose on bacterial growth.

As well as direct inhibition of bacterial growth by glucose (Additional file 1: Fig. S3), glucose affects bacteria via stimulation of plant responses via HKX1-related pathways. Leaf infiltration with glucose prior to bacterial exposure reduced disease symptoms and bacterial growth in WT Arabidopsis (Fig. 4). Initially, this effect was attributed to direct inhibition of bacterial growth by glucose. However, glucose induced callose deposition and earlier PR1 accumulationin plants (Fig. 5), indicating that additional pathways may be involved in the reduced disease phenotype. The impact of glucose exposure prior to infection was investigated with respect to growth of
Pto AvrRpm1 and Pto AvrRpt2 and in a hypersensitive response assay (Fig. 6). The enhanced disease resistance observed in WT plants pre-infiltrated with glucose was not apparent in $h x k 1$ mutant plants, further indicating that this phenomenon was not due primarily to the direct effects of glucose on bacterial cells. Taken together, these results indicate that glucose may activate HXK1-related pathways involved in mediating plant immune responses.

Based on our observations, we propose that HKX1 may be involved in several events simulated by glucose during the ETI response. The $h x k 1$ mutant would be expected to block the effect of glucose in ETI, because bacterial growth was similar with and without glucose upon Pto AvrRpt2 infiltration (Fig. 4b). However, faster HR also 


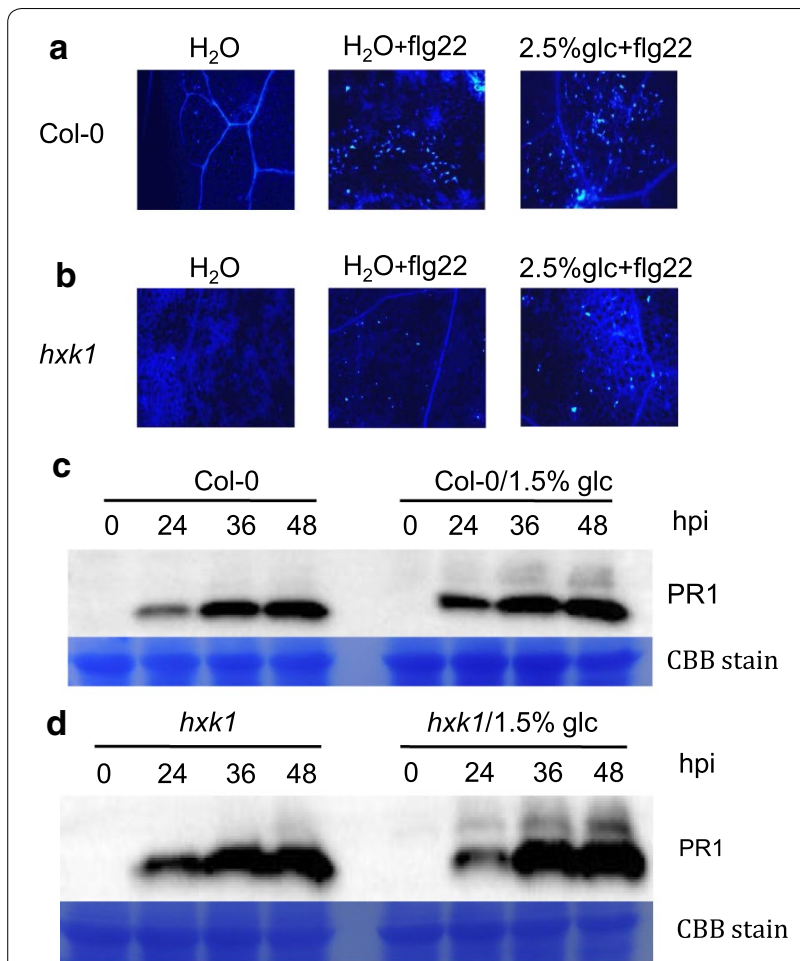

Fig. 5 Exogenous glucose positively enhances PTI. a Callose deposition in Col-0 leaves infiltrated with water or glucose ( $\mathrm{glc}$ ) $24 \mathrm{~h}$ before inoculating with $100 \mathrm{uM}$ flg22. b Callose deposition in $h \times k 1$ leaves as described in (a). c PR1 accumulation in Col-0 leaves infiltrated with glucose. $\mathbf{d}$ PR1 accumulation in $h \times k 1$ leaves infiltrated with glucose. Coomassie Brilliant Blue (CBB) staining was used to ensure equivalent loading. Experiments were repeated three times appeared in $h x k 1$ pre-infiltrated with glucose (Fig. 6d). Pto AvrRpt2 appears to be more glucose sensitive than Pto AvrRpm1 (Fig. 6a) which may explain why pre-infiltration still has an effect on HR in the $h x k 1$ mutant.

Our results indicate that HXK1 plays an important role in mediating the effects of glucose on plant immunity. HXK1 is active in plant immunity in both PTI and ETI, and also serves as a kinase that can phosphorylate glucose to glucose-6-phosphate. Absence of HXK1 in the $h x k 1$ mutant background led to higher bacterial growth, reduced callose deposition, and a delay in PR1 accumulation during PTI.

Glucose is ubiquitous in plant cells and is a good candidate as a signaling intermediary in plant immune responses. This study investigated the effects of glucose on plant defences. Exogenous glucose successfully reduced bacterial growth and disease symptoms. Low concentrations of exogenous glucose positively enhanced plant immunity via the PTI and ETI mechanisms. Glucose enhanced callose deposition, stimulated gene expression, and led to earlier PR1 accumulation, indicating involvement in the PTI pathway. Glucose also induced earlier cell death to prevent bacterial growth and earlier HR was observed. The effects of glucose on WT immune responses to infection were not observed in a $h x k 1$ mutant, indicating that exogenous glucose plays a role in plant immunity through HXK1-mediated pathways.
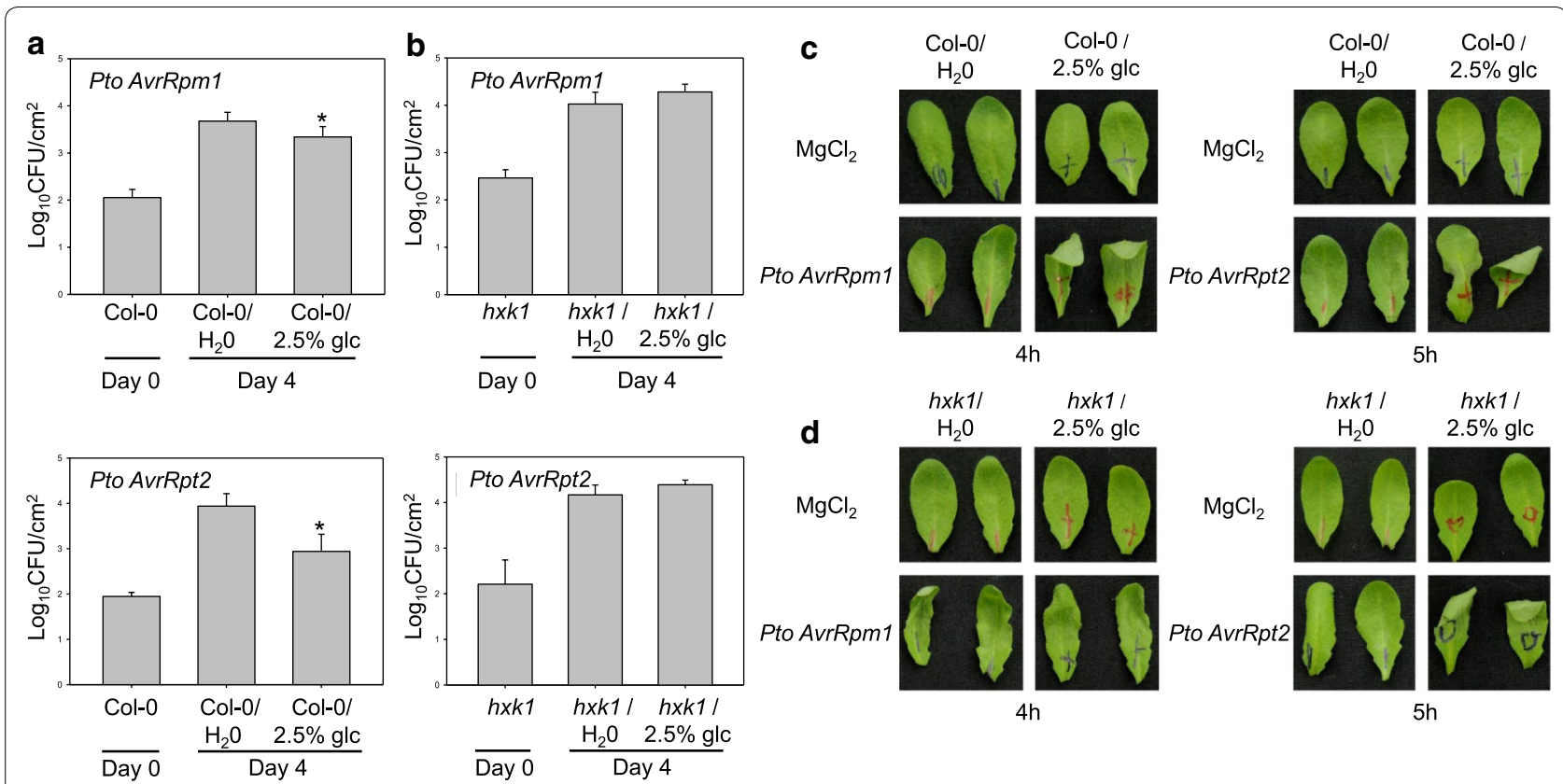

Fig. 6 Exogenous glucose positively enhances ETI. a Bacterial growth in Col-0 plants exposed to Pto AvrRpm 1 or Pto AvrRpt2 after pre-infiltration with glucose. b Bacterial growth in $h x k 1$ plants treated as described in a. c HR in Col-0 leaves exposed to Pto AvrRpm 1, Pto AvrRpt2, or MgCl 2 (control) after pre-infiltration with glucose or $\mathrm{H}_{2} \mathrm{O}$. $\mathbf{d} \mathrm{HR}$ in $h \times k 1$ leaves treated as described in $\mathbf{c}$. Error bars represent the standard error of the mean $(n=3)$. The asterisk indicates a statistically significant difference $(P<0.05$, Student's $t$-test). Similar results were obtained from three independent experiments 


\section{Supplementary information}

Supplementary information accompanies this paper at https://doi. org/10.1186/s13765-020-00560-8.

Additional file 1: Figure S1. HXK1 is not involved in RPT2-dependent suppression of Pto AvrRpt2. A. Bacterial accumulation in Col-0 and hxk1 after inoculation with $2 \times 10^{5} \mathrm{CFU} / \mathrm{ml}$ of Pto AvrRpt2. Error bars represent the standard error of the mean $(n=3)$. B. Ion leakage assay in $h \times k 1$ and WT leaves infiltrated with Pto AvrRpt2 or $\mathrm{MgCl}_{2}$ (control). C. Speed of HR in hxk1 and WT infiltrated with Pto AvrRpt2 or $\mathrm{MgCl}_{2}$ (control). Experiments were repeated three times with similar results. Figure S2. High concentrations of glucose damaged plants. A. Mature leaves of Col-0 and Ler plants infiltrated with $0,2.5,5,7.5$, and $10 \%$ glucose (glc) B. Arabidopsis Col-0 and Ler seedlings were grown at MS media containing $0 \%, 1.5 \%$ and $3 \%$ glucose. Experiments were conducted three times. Figure S3. Exogenous glucose suppressed bacterial growth in vitro. Growth of $P$. syringae strains Pto, Pto AvrRpm 1, and Pto AvrRpt2 in KB liquid media supplemented with different concentrations of glucose (glc) and $2 \times 10^{8} \mathrm{CFU} / \mathrm{ml}$ of bacteria Bacterial growth was measured at approximately $16 \mathrm{~h}$ after treatment.

\section{Acknowledgements}

This work was supported by grants from National Research Foundation of Korea (project no. NRF-2020R1/1A2073610) and by Next-Generation BioGreen21 Program (SSAC: PJ01326902) Rural Development Administration, Republic of Korea.

\section{Authors' contributions}

WJ, SU, RC, JYC and MGK wrote the manuscript with input from other authors. RC, SU, DMM, SOP, DTVN, HUK and GRR performed experiments. WYK and MGK edited the paper, gave support and conceptual advice. All authors discussed the contents and agreed on the contents of the paper and post no conflicting interest. All authors read and approved the final manuscript.

\section{Competing interests}

The authors declare no competing interests.

\section{Author details \\ ${ }^{1}$ College of Pharmacy and Research Institute of Pharmaceutical Science, PMBBRC, Gyeongsang National University, Jinju 660-701, Republic of Korea. ${ }^{2}$ Division of Applied Life Science (BK21+), PMBBRC, Gyeongsang National University, Jinju 660-701, Republic of Korea.}

Received: 6 August 2020 Accepted: 3 November 2020 Published online: 17 November 2020

\section{References}

1. Karve A, Rauh BL, Xia X, Kandasamy M, Meagher RB, Sheen J, Moore Bd (2008) Expression and evolutionary features of the hexokinase gene family in Arabidopsis. Planta 228:411-425

2. Moore B, Zhou L, Rolland F, Hall Q, Cheng WH, Liu YX, Hwang I, Jones T, Sheen J (2003) Role of the Arabidopsis glucose sensor HXK1 in nutrient, light, and hormonal signaling. Science 300:332-336

3. Zhou L, Jang JC, Jones TL, Sheen J (1998) Glucose and ethylene signal transduction crosstalk revealed by an Arabidopsis glucose-insensitive mutant. Proc Natl Acad Sci USA 95:10294-10299

4. Sarowar S, Lee JY, Ahn ER, Pai HS (2008) A role of hexokinases in plant resistance to oxidative stress and pathogen infection. J Plant Biol 51:341-346

5. Jang JC, León P, Zhou L, Sheen J (1997) Hexokinase as a sugar sensor in higher plants. Plant Cell 9:5-19

6. Jones JDG, Dangl JL (2006) The plant immune system. Nature 444:323-329

7. Zipfel C (2009) Early molecular events in PAMP-triggered immunity. Curr Opin Plant Biol 12:414-420
8. Gómez-Gómez L, Boller T (2000) FLS2: An LRR receptor-like kinase involved in the perception of the bacterial elicitor flagellin in Arabidopsis. Mol Cell 5:1003-1011

9. Zipfel C, Kunze G, Chinchilla D, Caniard A, Jones JDG, Boller T, Felix G (2006) Perception of the bacterial PAMP EF-Tu by the receptor EFR restricts agrobacterium-mediated transformation. Cell 125:749-760

10. Chinchilla D, Zipfel C, Robatzek S, Kemmerling B, Nürnberger T, Jones JDG, Boller T (2007) A flagellin-induced complex of the receptor FLS2 and BAK1 initiates plant defence. Nature 448:497-500

11. Heese A, Hann DR, Gimenez-lbanez S, Jones AME, He K, Li J, Rathjen JP (2007) The receptor-like kinase SERK3/BAK1 is a central regulator of innate immunity in plants. Proc Natl Acad Sci USA 104:12217-12222

12. Boller T, Felix G (2009) A renaissance of elicitors: perception of microbeassociated molecular patterns and danger signals by pattern-recognition receptors. Annu Rev Plant Biol 60:379-406

13. Gao QM, Zhu S, Kachroo P, Kachroo A (2015) Signal regulators of systemic acquired resistance. Front Plant Sci 6:228

14. Xin XF, He SY (2013) Pseudomonas syringae pv. tomato DC3000: A Model Pathogen for Probing Disease Susceptibility and Hormone Signaling in Plants. Annu Rev Phytopathol 51:473-498

15. Dodds PN, Lawrence GJ, Catanzariti AM, Teh T, Wang CIA, Ayliffe MA, Ellis JG (2006) Direct protein interaction underlies gene-for-gene specificity and coevolution of the flax resistance genes and flax rust avirulence genes. Proc Natl Acad Sci USA 103:8888-8893

16. Krasileva KV, Dahlbeck D, Staskawicz BJ (2010) Activation of an Arabidopsis resistance protein is specified by the in planta association of its leucine-rich repeat domain with the cognate oomycete effector. Plant Cell 22:2444-2458

17. Mackey D, Belkhadir Y, Alonso JM, Ecker JR, Dangl JL (2003) Arabidopsis RIN4 is a target of the type III virulence effector AvrRpt2 and moculates RPS2-mediated resistance. Cell 112:379-389

18. Mackey D, Holt BF III, Wiig A, Dangl JL (2002) RIN4 interacts with Pseudomonas syringae Type III effector molecules and is required for RPM1mediated resistance in Arabidopsis. Cell 108:743-754

19. Chisholm ST, Coakerm G, Day B, Staskawicz BJ (2006) Host-microbe interactions: shaping the evolution of the plant immune response. Cell 124:803-814

20. Axtell MJ, Staskawicz BJ (2003) Initiation of RPS2-specified disease resistance in Arabidopsis is coupled to the AvrRpt2-directed elimination of RIN4. Cell 112:369-377

21. Boyes D, Nam J, Dangl JL (1998) The Arabidopsis thaliana RPM1 disease resistance gene product is a peripheral plasma membrane protein that is degraded. Proc Natl Acad Sci USA 95:15849-15854

22. Liu J, Elmore JM, Lin ZD, Coaker G (2012) A receptor-like cytoplasmic kinase phosphorylates the host target RIN4, leading to the activation of a plant innate immune receptor. Cell Host Microbe 9:137-146

23. Chisholm ST, Dahlbeck D, Krishnamurthy N, Day B, Sjolander K, Staskawicz BJ (2005) Molecular characterization of proteolytic cleavage sites of the Pseudomonas syringae effector AvrRpt2. Proc Natl Acad Sci USA 102:2087-2092

24. Axtell MJ, Chisholm ST, Dahlbeck D, Staskawicz BJ (2003) Genetic and molecular evidence that the Pseudomonas syringae type III effector protein AvrRpt2 is a cysteine protease. Mol Microbiol 49:1537-1546

25. Uddin MN, Akhter S, Chakraborty R, Baek JH, Cha JY, Kang PSJ, H, Kim WY, Lee SY, Mackey D, Kim, MG, (2017) SDE5, a putative RNA export protein, participates in plant innate immunity through a flagellin-dependent signaling pathway in Arabidopsis. Sci Rep 7:1-11

26. Kim MG, Cunha LD, McFall AJ, Belkhadir Y, DebRoy S, Dangl JL, Mackey D (2005) Two Pseudomonas syringae type III effectors inhibit RIN4-regulated basal defense in Arabidopsis. Cell 121:749-759

27. Buell CR, Joardar V, Lindeberg M, Selengut J, Paulsen IT, Gwinn ML, Dodson RJ, Deboy DAS, Kolonay JF, Madupu R, Daugherty S, Brinkac L, Mj B, Haft DH, Nelson WC, Davidsen T, Zafar N, Zhou L, Liu J, Yuan Q, Khouri H, Fedorova N, Tran B, Russell D, Berry K, Utterback T, Aken SEV, Feldblyum TV, D'Ascenzo M, Deng WL, Ramos AR, Alfano JR, Cartinhour S, Chatterjee AK, Delaney TP, Lazarowitz SG, Martin GB, Schneider DJ, TangCollmer A (2003) The complete genome sequence of the Arabidopsis and tomato pathogen Pseudomonas syringae pv. tomato DC3000. Proc Natl Acad Sci USA 100:10181-10186 
28. Asai T, Tena G, Plotnikova J, Willmann MR, Chiu WL, Gomez-Gomez L, Boller T, Ausubel FM, Sheen J (2002) Map kinase signalling cascade in Arabidopsis innate immunity. Nature 415:977-983

29. Hauck P, Thilmony R, He SY (2003) A Pseudomonas syringae type III effector suppresses cell wall-based extracellular defense in susceptible Arabidopsis plants. Proc Natl Acad Sci USA 100:8577-8582

30. Bestwick CS, Bennett MH, Mansfield JW (1995) Hrp mutant of Pseudomonas syringae pv phaseolicola induces cell wall alterations but not membrane damage leading to the hypersensitive reaction in lettuce. Plant Physiol 108:503-516

31. Nowicki M, Nowakowska M, Kłosińska U, Golik P, Kozik EU, Lichocka M (2012) A simple dual stain for detailed investigations of plant-fungal pathogen interactions. Veg Crop Res Bull 77:61-74

32. Ham JH, Kim MG, Lee SY, Mackey D (2007) Layered basal defenses underlie non-host resistance of Arabidopsis to Pseudomonas syringae pv. phaseolicola. Plant J 51:604-616

33. Ray SK, Macoy DM, Kim WY, Lee SY, Kim MG (2019) Role of RIN4 in regulating PAMP-triggered immunity and effector-triggered immunity: current status and future perspectives. Mol Cells 42:503
34. Rivière MP, Marais A, Ponchet M, Willats W, Galiana E (2008) Silencing of acidic pathogenesis-related PR-1 genes increases extracellular $\beta-(1 \rightarrow 3)$ glucanase activity at the onset of tobacco defence reactions. J Exp Bot 59:1225-1239

35. Olivares-Marin IK, González-Hernández JC, Regalado-Gonzalez C, Madrigal-Perez LA (2018) Saccharomyces cerevisiae exponential growth kinetics in batch culture to analyze respiratory and fermentative metabolism. J Vis Exp 139:1-10

36. Lu J, Carter DA, Turnbull L, Rosendale D, Hedderley D, Stephens J, Gannabathula S, Steinhorn G, Schlothauer RC, Whitchurch CB, Harry EJ (2013) The effect of New Zealand Kanuka, Manuka and clover honeys on bacterial growth dynamics and cellular morphology varies according to the species. PLOS ONE 8:e55898

\section{Publisher's Note}

Springer Nature remains neutral with regard to jurisdictional claims in published maps and institutional affiliations.

\section{Submit your manuscript to a SpringerOpen ${ }^{\circ}$ journal and benefit from:}

- Convenient online submission

- Rigorous peer review

- Open access: articles freely available online

- High visibility within the field

- Retaining the copyright to your article

Submit your next manuscript at $\gg$ springeropen.com 